\title{
Testing System for Unmanned Aerial Vehicles Microelectromechanical Sensors
}

\author{
M. P. Vasylenko \\ Aviation Computer Integrated Complexes department \\ National Aviation University \\ Kyiv, Ukraine \\ vasylenkom.89@gmail.com
}

\begin{abstract}
Designed system for microelectromechanical sensors testing before use them in the unmanned aerial vehicles navigation systems. Microelectromechanical sensors pressure and temperature sensor, magnetic field sensor and inertial unit were studied. Experimental results shows that designed testing equipment allows to calibrate microelectromechanical sensors without necessity of performing test flights that reduces the risk of unmanned aerial vehicles damaging in case of significant measurement errors.
\end{abstract}

Keywords-microelectromechanical sensors; calibration; unmanned aerial vehicles; navigation

\section{INTRODUCTION}

Nowadays unmanned aerial vehicles (UAVs) are widely used in different areas of life. They vary greatly by size, action range, load capacity, flight time, etc. Small personal UAVs usually have action range up to $300 \mathrm{~m}$ so can be easily controlled by visual determination of their attitude and position. Unmanned aerial vehicles with several kilometers action range must have instruments to determine aircrafts altitude, speed, attitude and coordinates to give the operator necessary information about flight through the telemetry channel. Advanced UAV systems must have autopilot that at least allows UAV to return in case of ground control station signal loss. The most advanced UAVs must have ability to perform the autonomous flight by the predefined route. But all these UAV types must have instruments to determine the main flight navigation parameters.

\section{Problem Statement}

Determination of these parameters requires special measurement instruments such as altimeters, gyroscopes, accelerometers, etc and the more instruments you have the higher accuracy is. But each of them has some mass and energy consumption that effects UAVs load capacity, flight time and range. So it is necessary to have as accurate and lightweight instruments with as low energy consumption as possible.

This problem can be solved by use of microelectromechanical (MEMS) sensors which are small, have high accuracy and sensitivity, low energy consumption and digital output [1].
But MEMS sensors also have errors that must reduced or removed. Such calibration can be done by the results of test flight or laboratory tests that are much faster and safer because can not result in UAV damaging or loss.

So it is necessary to have the set of laboratory equipment to perform such tests.

\section{TESTING EQUIPMENT}

Set of equipment must include instruments to test barometric, inertial and magnetic field sensors. Schematic diagram of designed system is shown in Fig. 1.

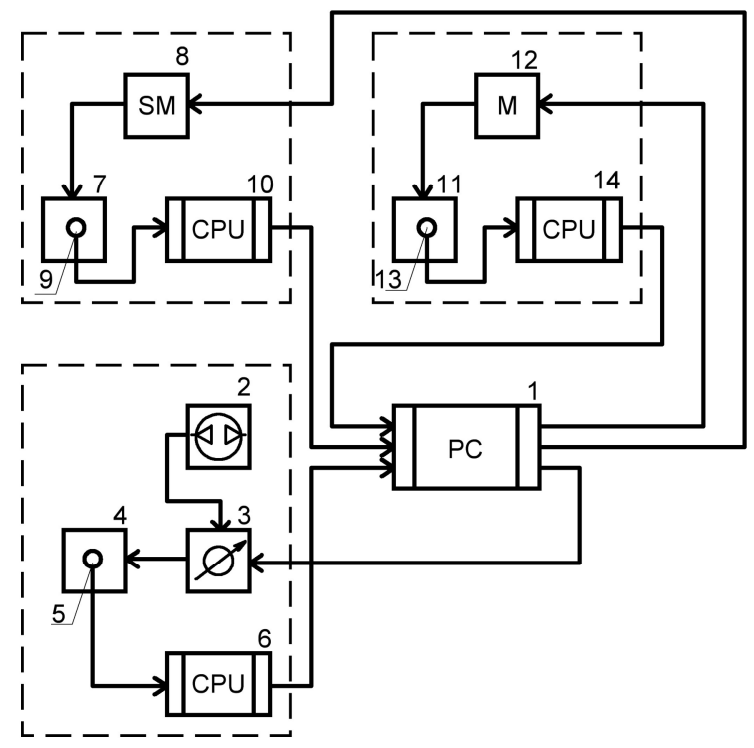

Fig. 1. Structure of MEMS sensors testing system.

Systems includes computer 1 that gathers information from tested barometric sensor 5, magnetic field sensor and accelerometer 9 and gyroscope 13 and controls the testing equipment that includes pneumatic pump 2, pressure adjustment device 3 , pressurized case 4 , stabilized platform 7 with servomotors 8, turntable 11 with motor 12 . Microprocessors 6, 10 and 14 are used to provide the reading, processing and transmission of raw data from tested sensors. 
Computer software allows to form the reference values of measured parameters for each sensors, compares them with measured values and calculates errors. In case, when error exceeds value stated in the sensor specifications - calibration coefficients are calculated.

These coefficients are used to eliminate or reduce the error values. Designed system was used to test GY-80 measurement unit that includes BMP180 pressure sensor, HMC5883L magnetic field sensor, and MPU-6050 inertial measuring unit that consists of triple-axis accelerometer and triple-axis gyroscope.

\section{BAROMETRIC MEMS SENSORS}

Microelectromechanical sensors pressure sensors use tenso- and piezoresistive effects.

Tensoresistive effect is the change of interatomic distances during the sensing elements deformation that causes the change of crystals energy zones and changes in electrical charge carriers concentration, effective mass, redistribution between energy maximums in conductance zone and minimums in valence zone.

Piezoresistive sensors are monocrystalline silicic devices which use monocrystalline silicic membrane as the sensitive element and piezoresistors, formed by the diffusion method, which are connected into the Wheatstone bridge. Sensitive element of such sensor is crystal placed on the dielectric base which can deform under the action of pressure that causes the resistance change and unbalance of Wheatstone bridge that is linearly dependent from the deformation.

Construction of MEMS pressure sensor sensitive element [1] is shown in Fig. 2.

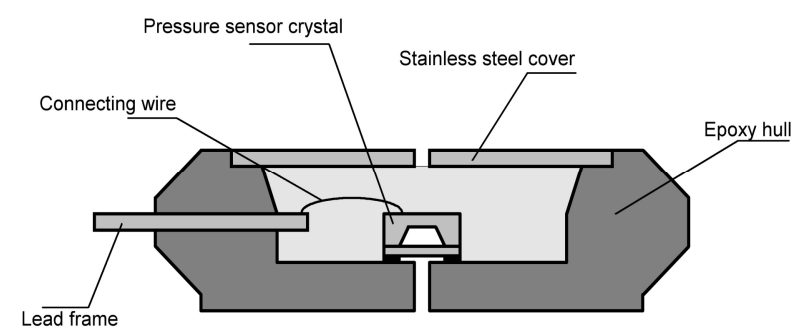

Fig. 2. Absolute pressure sensor sensitive element construction.

Main element of pressure sensor is crystal with silicic diaphragm with sensitive piezoresistive chain on it. Inner space of the casing is filled with silicon gel that isolates the sensitive crystal and joints but allows pressure to effect the diaphragm. It is necessary for the defense from the harmful environmental influences.

Pressure sensing crystal is placed on silicic crystal holder that is glued to the sensors casing. Plastic casing has one or two pressure ports and stainless steel cover.

The tested sample of pressure sensor is BMP180 that consists of sensitive element, power stabilizer, temperature compensation scheme, ADC and EEPROM. Structure of BMP180 is shown in Fig. 3.

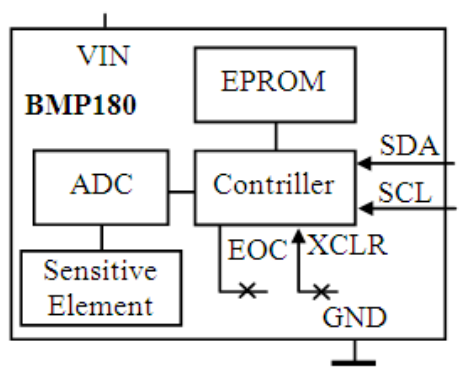

Fig. 3. Structure of BMP180 pressure sensor.

Sensor can also give information about the environment temperature. EEPROM includes calibration coefficients for thermal compensation system.

After the initialization BMP180 controller reads calibration coefficients from EEPROM and starts to read raw data from pressure and temperature sensors in loop cycle through the I2C interface and by the predefined algorithm calculates true values of pressure and temperature.

Specifications of BMP180 are shown in Table I.

TABLE I. BMP180 SPECIFICATIONS

\begin{tabular}{|l|c|}
\hline \multicolumn{1}{|c|}{ Parameter } & Value \\
\hline Atmospheric pressure measurement range & $\begin{array}{c}300 \ldots 1100 \mathrm{hPa} \\
(225 \ldots 825 \mathrm{~mm} . \mathrm{hg} .)\end{array}$ \\
\hline Pressure measurement accuracy & $\pm 3 \mathrm{~Pa}(17 \mathrm{~cm})$ \\
\hline Temperature measurement range & $0 \ldots 65^{\circ} \mathrm{C}$ \\
\hline Temperature measurement accuracy & $\pm 0.5 \ldots 1^{\circ} \mathrm{C}$ \\
\hline
\end{tabular}

Experiments show altitude measurement error $\pm 15 \mathrm{~cm}$ that is much lower than for traditional barometric equipment.

\section{MEMS MAGNETIC FiELD SENSORS}

Earth magnetic field (EMF) can be approximated by dipole model shown in Fig. 3. Magnetic axis makes 11.5 degree angle with the Earth rotation axis. Magnetic field lines directed almost vertically in the centers of northern and southern hemispheres and horizontal at the equator and point at the North magnetic pole.

Main characteristic of EMF is its strength $T$ that is the amount and direction of force acting the mass at the current point of the Earth. Direction of EMF strength vector is determined by three components directed along the $X, Y$ and $Z$ axis of rectangular coordinate system (Fig. 4).
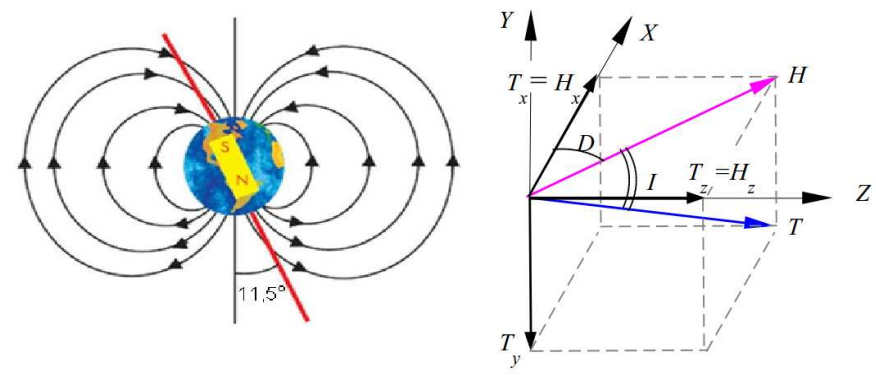

Fig. 4. Dipole model of Earth magnetic field. 
These are horizontal component of magnetic field strength $H$, magnetic declination $D$ (angle between $H$ and plane of geographical meridian) and magnetic inclination $I$ (angle between $T$ and plane of horizon). Direction of EMF strength horizontal component $H$ is called the direction of magnetic meridian and used to determine heading in navigation systems.

Existing airborne navigation systems determine magnetic heading using inductive compasses that have big size, mass and errors caused by the float mount.

In order to build electronic compass based on the Hall effect or magnetoresistance effect were developed compass kits based on the complex of highly sensitive magnetic field sensors and MEMS-accelerometers to determine the horizontal position controlled by the microprocessor system [2]. Accelerometer is used as an inclinometer and measures pitch and roll angles which are also used by the microprocessor to determine the magnetic heading.

One of such kits we used as the test sample is Honeywell HMC5883L with specifications given in Table II.

TABLE II. HMC5883L SPECIFICATIONS

\begin{tabular}{|l|c|}
\hline \multicolumn{1}{|c|}{ Parameter } & Value \\
\hline Measurement range & $-8 \ldots+8 \mathrm{Gs}$ \\
\hline Measurement error & $\pm 230 \mathrm{mGs}$ \\
\hline Nonlinearity & $0.1 \%$ \\
\hline Operating voltage & $2.16 \ldots 3.6 \mathrm{~V}$ \\
\hline Operating current & $0.1 \mathrm{~mA}$ \\
\hline
\end{tabular}

Experimental results show that HMC5883L provides magnetic heading measurement with maximum error $1 \ldots 2$ degrees with digital output via I2C interface that is suitable for use with any modern controller or UAV autopilot.

Magnetic field sensor consists of sensing element and electronic measuring block. Principle of action is based on the changes in electrical resistance of ferromagnetic material under the action of external magnetic field. Electrical current through such element changes with non-linear law so linearisation is performed by the sensor itself.

Magnetometer structure is shown in Fig. 5. The basis of such system is measurement unit. Information about the current azimuth is transmitted to the converter. Due to high sensitivity of measuring unit residual magnetization related to the inner structure may appear.

Residual magnetization can be removed by the demagnetizing pulse from SET / RESET coil (Fig. 5).

Converter is an ADC that transforms signals digitalization and controls low frequency measurements. Next digital signal is transmitted to the protocol forming device that is based on microcontroller that performs coordination and forms data for further processing. Information about current heading is transmitted to the conjudation device where it is converted into the serial cod and transmitted to the output device.

Vehicles own magnetic field may cause errors and distorts the magnetic field distribution (Fig. 6a) by moving it's center and transforming it into ellipse.

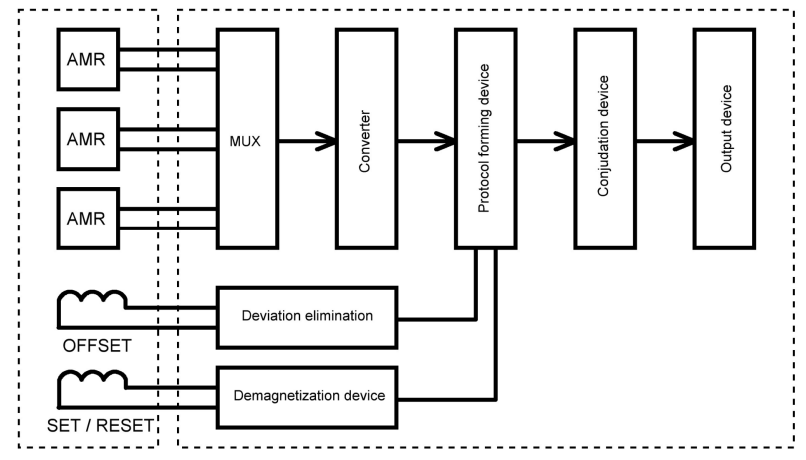

Fig. 5. Structure of HMC5883L magnetic field sensor.

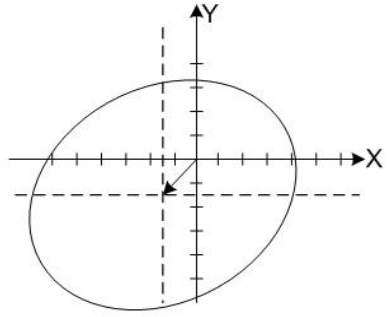

(a)

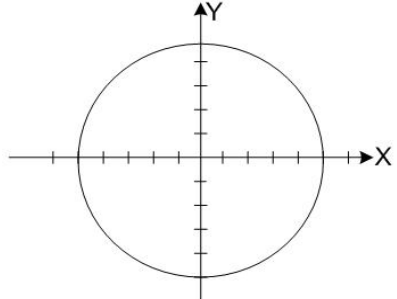

(b)
Fig. 6. Magnetic field distribution.

To remove such deviation the OFFSET coil is used (see Fig. 5). The coil is placed near the AMR bridge and has strictly defined geometry. Vehicle magnetic field and coil generated magnetic field are added with the account of sign and considered as one field. Magnetic field distribution correction is performed by calculation of two correction values by the predefined algorithm in order to obtain the circular distribution (see Fig. 6b).

\section{MEMS INERTIAL SENSORS}

Advances in MEMS technology development allowed to make inertial sensors much smaller and cheaper that increased the demand for such sensors in different areas from industrial, transport and military applications to customer devices and entertainment industry. Manufactures started to produce complex inertial measuring units (IMU) which contain tripleaxis gyroscope and accelerometer.

For laboratory tests MPU-6050 was used. Structure of tested IMU is shown in Fig. 7.

It contains digital motion processor (DMP), integrated triple-axis accelerometer piezoelectric, triple-axis vibration gyroscope and temperature sensor with I2C digital output.

The schematic diagram of the piezoelectric accelerometer is shown in Fig. 8. The crystal of the piezoelectric [3] - [4] material is combined with the inertial mass, and the electrical contact of the piezoelectric element in the system is carried out by means of two strain gauges located on each side of the crystal.

Acceleration acting on the sensor leads to a difference in movement of the casing and the inertial mass, resulting in a force deforming the crystal of the piezoelectric material. There 
is a redistribution of charges on both sides of the crystal, while the output signal of the accelerometer is directly proportional to the acceleration or the level of vibrations. By means of electrodes, changes in the system are transferred to the control unit.

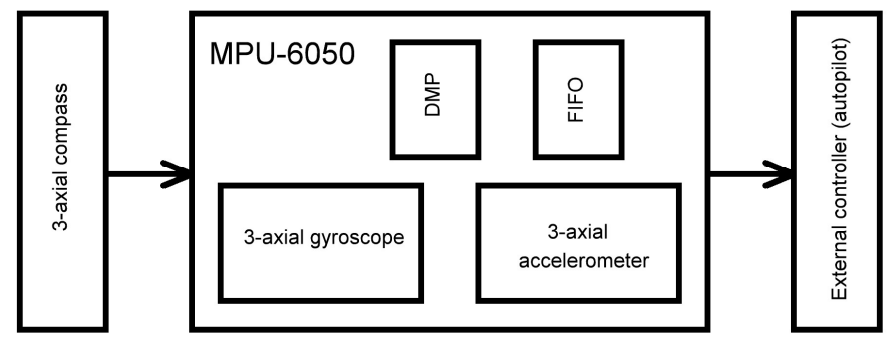

Fig. 7. Structure of MPU-6050 inertial measuring unit.

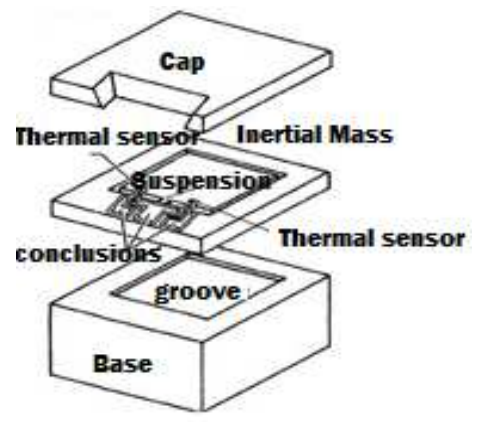

Fig. 8. Schematic diagram of piezoelectric accelerometer.

Vibration gyroscope is a metal disc with $1 \mathrm{~mm}$ diameter rigidly fixed in the center of silicon surface [5] with the electrodes connected to the outer rim. Under the action of the voltage applied to the electrodes the main oscillation mode is generated. For example, if mode is generated along the $X$ axis - disc expands along the $X$ axis and squeezes along the $Y$ axis as shown in Fig. 9.

If disc starts to turn around $Z$ axis Coriolis force tries to cause the oscillations along the $Y$ axis. Second measuring mode appears and main axis of ellipse turns 45 degrees. Amplitude of these oscillations is proportional to the angular speed of rotation.

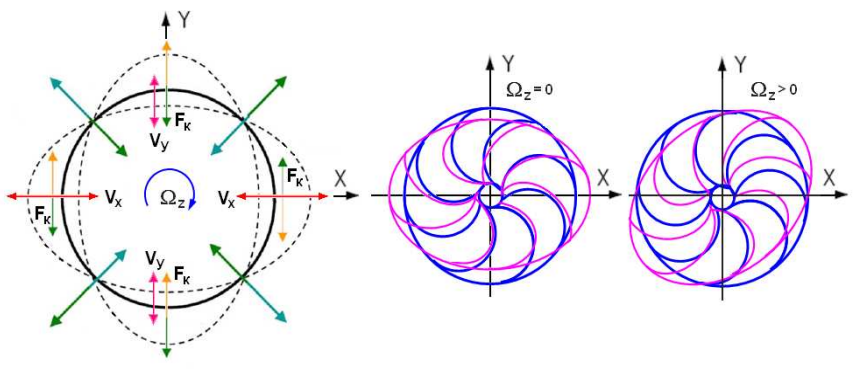

Fig. 9. Action of vibration gyroscope.
Specifications of IMU are shown in Table III.

Integrated DMP of IMU has algorithms of nine-axis data detection which can be used in case of external magnetic compass connection and provides some inertial navigation algorithms (rotation matrices, quaternions, Euler angles). Nine-axis detection includes software data combination, sensor control and calibration, gyroscope and accelerometer data filtering.

TABLE III. MPU-6050 SPECIFICATIONS

\begin{tabular}{|l|c|}
\hline \multicolumn{1}{|c|}{ Parameter } & Value \\
\hline Gyroscope measurement range & $\begin{array}{c} \pm 250 ; \pm 500 ; \pm 1000 ; \\
\pm 2000 \mathrm{deg} / \mathrm{s}\end{array}$ \\
\hline Accelerometer measurement range & $\pm 2 ; \pm 4 ; \pm 8 ; \pm 16 \mathrm{~g}$ \\
\hline Operating voltage & $3.7-5.5 \mathrm{~V}$ \\
\hline Operating current & $10 \mathrm{~mA}$ \\
\hline
\end{tabular}

\section{CONCLUSIONS}

Latest advances in MEMS technologies development allows to make sensors extremely small and lightweight. For example, GY-80 sensor assembly that includes triple-axis accelerometer, triple-axis gyroscope, magnetic compass and pressure sensor has size $25.8 \times 16.8 \mathrm{~mm}$ that is much smaller than the classical equipment. It allows to make UAV significantly lighter and carry more useful payload depending on the tasks UAV is designed for.

Microelectromechanical sensors have low energy consumption and energy saving modes which allow to save power in critical situations decreasing the chance of UAV loss.

Designed testing system allows to determine MEMS sensors errors, calculate calibration coefficients and remove the sensors with malfunctions without performing test flights that makes testing process much faster, easier and safer.

\section{REFERENCES}

M.A. Huff, Position Paper: MEMS Manufacturing . The MEMS Exchange: Reston, Virginia, U.S.A., 1999.

J.J. Allen, R.D. Kinney, J. Sarsfield, M.R. Daily, J.R. Ellis, J.H. Smith, S. Montague, R.T. Howe, B.E. Boser, R. Horowitz, A.P. Pisano, M.A. Lemkin, W.A. Clark, and C.T. Juneau, Integrated Micro-Electro Mechanical Sensor Development for Inertial Application. Sandia National Laboratories: Albuquerque, NM, U.S.A., 1998.

M. Park, and Y. Gao, Error Analysis of Low-Cost MEMS based Accelerometers for Land Vehicle Navigation. Presented at ION GPS2003, Portland, UT, U.S.A., 2002.

C. Elster, A. Link, and T. Bruns, "Analysis of dynamic measurements and determination of time-dependent measurement uncertainty using a second-order model", Meas. Sci. Technol., 18, (2007), p. 3682-3687.

A. Link, A. Täubner, W. Wabinski, T. Bruns, and C. Elster, "Calibration of accelerometers: determination of amplitude and phase response upon shock excitation", Meas. Sci. Techn., 17, (2006), p. 1888-1894. 